\title{
Research on a novel magnetic tilt sensor designed using Hall elements and ferrofluid
}

\author{
Yavuz Öztürk* ${ }^{*}$ Ismail Yariçî***
}

\begin{abstract}
In this study, a simple, adjustable, bidirectional tilt sensor was designed using a pair of linear Hall effect sensors and magnets. Theoretical analysis and experimental results of the sensor system were presented. The working principle of the designed sensor is based on sensing the magnetic field of a mobile magnet which displaces with respect to the tilt angle. Two magnet sets were placed at the two ends of the system to apply repulsive restoring forces on the mobile magnet. The mobile magnet was coated with a light hydrocarbon based ferrofluid as a lubricant to reduce friction. Fixed Hall effect sensors were placed face to face at the two sides of the mobile magnet to monitor the magnetic field of the mobile magnet. It was shown that both experimentally and theoretically, it is possible to measure the approximate tilt angle linearly and quadratically by calculating the sum and difference of the Hall sensor voltages for the relatively small movements of the mobile magnet. Moreover, the system was also examined for the different sets of side magnets. For three different side magnet configurations, approximately $0.7,1.1$ and $1.68 \mathrm{~V} / \mathrm{rad}$ sensitivity values were observed in the linear range.
\end{abstract}

K e y w or d s: Hall sensor, inclinometer, bi-directional tilt sensor, magnetic fluid, magnetic field measurement

\section{Introduction}

The tilt sensors have been demanded in many industrial applications from robotics to construction and have a wide range of uses. Due to this widespread application area, tilt sensors with different operating principles have been studied both theoretically and experimentally. Research on the magnetic, inductive, capacitive and optical types of tilt sensors has been reported in the literature [1-6]. Furthermore, there has been some research on the magnetic and inductive type tilt sensors designed by use of magnetic fluid and magnets [2-4].

Magnetic fluids mainly consist of dispersed magnetic surfactant coated nanoparticles and a carrier liquid. Nano-magnetic fluids generally show super-paramagnetic properties and when used with magnets, they exhibit properties such as lubrication and sealing, depending on the carrier fluid. One of the reasons for the use of magnetic fluid in the tilt sensor is that the magnetic fluid moves along with the magnet and reduces friction $[4,7,8]$. In several studies on tilt sensors, the windings are used as sensing elements based on the Faraday law [2-4]. As an alternative sensing element Hall effect sensors have several advantages such as wide range of constant or varying fields measurement capability, high repeatability and low power $(0.1-0.2 \mathrm{~W})$ [1]. To exploit these advantages, Hall effect sensors are also used in tilt sensors with various configurations [9-11]. In an earlier research we found, which is similar to our study, using a single Hall effect sensor measurement range up to 15 degrees was examined, and no restoring side magnets were used [9].
In the present study, an alternative tilt sensor design with the advantages of simplicity, adjustable sensitivity and bidirectional measurement of tilt angle was presented. In the proposed system, a mobile magnet placed in a non-magnetic tube was balanced with the two side magnet sets placed at the ends. Two Hall effect sensors were used to probe magnetic field changes due to the displacement of a ferrofluid coated mobile magnet in relation to inclination. The system dynamics were investigated both experimentally and theoretically. The sum and difference of the Hall sensor voltages, calculated by using the Taylor series, and their linear and quadratic dependence to the tilt angle was shown respectively. Furthermore, the effect of the different sets of side magnets was also investigated experimentally.

\section{The tilt sensor system}

The system was designed with a magnetic fluid coated mobile magnet in a tube (Magnet-0), two Hall effect magnetic field sensors and two side magnet sets (S.Mag.-1 and S.Mag.-2) as shown in Fig. 1. The mobile and ferrofluid coated Magnet-0 was placed between two Hall effect sensors and two side magnets which were fixed at the end of the tube. The polarity of the magnets was arranged to apply force to each other repulsively. The linear Hall effect sensor provides a voltage output proportional to the applied magnetic field which can be either positive or negative. The voltage output of sensors we used has a positive null voltage at zero magnetic field and this value increases when a positive magnetic field is sensed and

*Department of Electrical and Electronics Engineering, Ege University, 35100, Izmir, Turkey, **Department of Electrical and Electronics Engineering, Aydın Adnan Menderes University, 09100, Aydın, Turkey, yavuz.ozturk@ege.edu.tr 


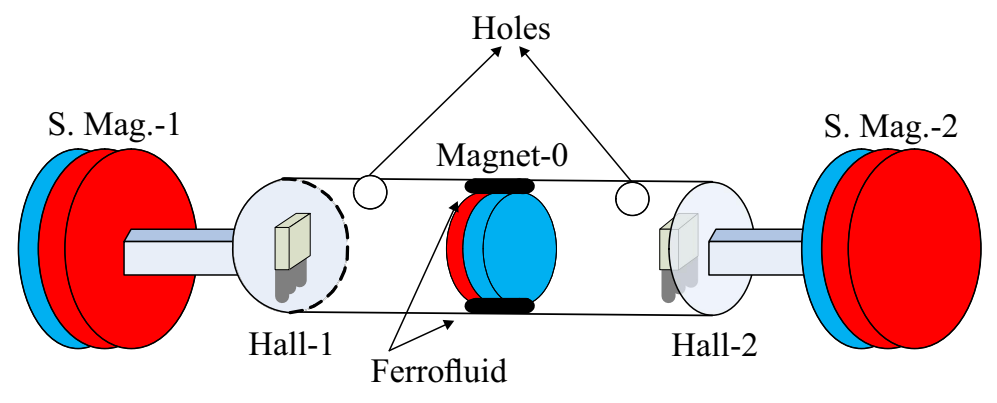

Fig. 1. The diagram of designed tilt sensor

decreases when a negative magnetic field is sensed. The magnetic field value will increase on the Hall sensors when the mobile magnet is put close up to any one of them and vice versa. As a result of this magnetic field change, the angle and direction of inclination can be determined by using the system shown in Fig. 1.

The advantages of using ferrofluid as a lubricant around the magnet are threefold: (i) it reduces the friction and moves with the magnet, (ii) it decreases the response time of the magnet to the external fields, and (iii) it increases the sensitivity. Further, the ferrofluid completely covers the magnet in the non-magnetic tube and seals the sides of magnet-0, which makes it harder to move the magnet because of air compression. Therefore, the air holes are opened in order to eliminate this problem. Another usage of these holes is that when the magnet reaches the level of the hole, the magnetic fluid plugs this hole and prevents the magnet from moving beyond the hole. As an alternative solution, it is possible to solve the sealing effect by using a magnet with a hole in the center.

All magnets used in the system are commercial neodymium magnets. A pair of AH49E model linear Hall effect sensors with the specifications of a $0-0.1 \mathrm{~T}$ measuring range and $1.6 \pm 0.5 \mathrm{mV} / \mathrm{G}$ sensitivity were used in the system. These sensors were operated by a $5 \mathrm{~V}$ DC-supply voltage. They feature a null voltage output of around $50 \%$ of the supply voltage when there is no magnetic field and provide a voltage output that is linearly proportional to the applied magnetic field. The linear output range of the Hall sensors is around $\pm 0.1 \mathrm{~T}$ which corresponds to the voltage output of around between $0.9 \mathrm{~V}$ to $4.1 \mathrm{~V}$. The sensor output voltages were measured with the Brymen BM257 model multimeters.

A commercial EFH-1 magnetic fluid was used as a lubricant around magnet-0. EFH-1 ferrofluid consists of nano-sized particles and liquids of light hydrocarbon as a carrier. The saturation magnetization $\left(M_{\mathrm{S}}\right)$ and density of this ferrofluid were $44 \mathrm{mT}$ at $25^{\circ} \mathrm{C}$ and $1.21 \times$ $10^{3} \mathrm{~kg} / \mathrm{m}^{3}$ respectively. Each magnet of the side magnet sets (S.Mag.-1 and S.Mag.-2) is $20 \mathrm{~mm}$ in diameter and $2 \mathrm{~mm}$ in length. Magnet- 0 is $12 \mathrm{~mm}$ in diameter and $10 \mathrm{~mm}$ in length. The selected tube inner radius is $12.7 \mathrm{~mm}$ in diameter and the distance between the holes is set to $27.5 \mathrm{~mm}$. The distance between the Hall effect sensors is $47 \mathrm{~mm}$ and the distance between the side magnets is $117 \mathrm{~mm}$. The distance between each hole and Hall sensor was determined by considering the linear range of the Hall sensors, which was not exceeded in our measurements. The system was mounted on a Thorlabs PR01/M model manual rotational stage to adjust the tilt angle.

\section{Theoretical analysis}

The Lorentzian approach is one of the methods to calculate the magnetic field of a magnet. In this approach, the magnetization $M$ of uniformly magnetized magnets is assumed to be equivalent to the surface current density. By using the Lorentzian approach, the magnetic field of a uniformly magnetized solid cylindrical magnet (Fig. 1: Magnet-1) with a length of $a$ and radius of $r$ can be calculated on its axis $(z)$ like a finite solenoid with the surface current of $M$

$$
B_{z}(z)=\frac{\mu_{0} M}{2}\left(\frac{z}{\sqrt{r^{2}+z^{2}}}+\frac{a-z}{\sqrt{\left(r^{2}+(a-z)^{2}\right.}}\right)
$$

where, $\mu_{0}$ is the permeability of free space. There are several studies in which approximate solutions of (1) were obtained. In these studies, it was shown that the magnetic field is proportional to the power law of distance as $z^{-3}$ and the magnetic force is proportional to $z^{-4}$ when $a \gg z[12,13]$.

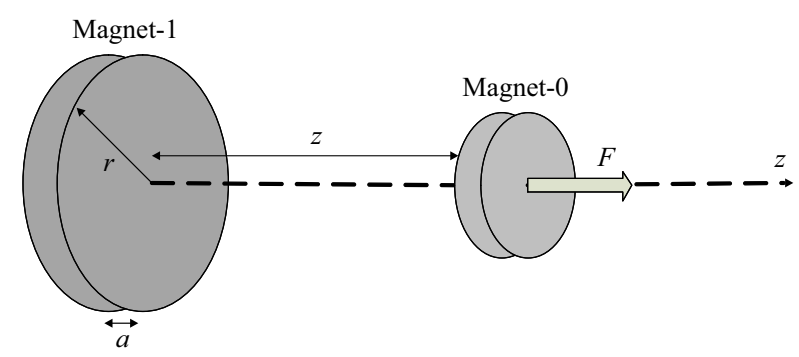

Fig. 2. Schematics of repelling magnets

Also, there are several articles where both the field and the force between the two magnets are examined theoretically by using relatively complex mathematical solutions $[12,14]$. For the sake of simplicity, a practical expression of force and magnetic field were calculated analytically with some assumptions and approximations in the following part. It is assumed that the magnetic 


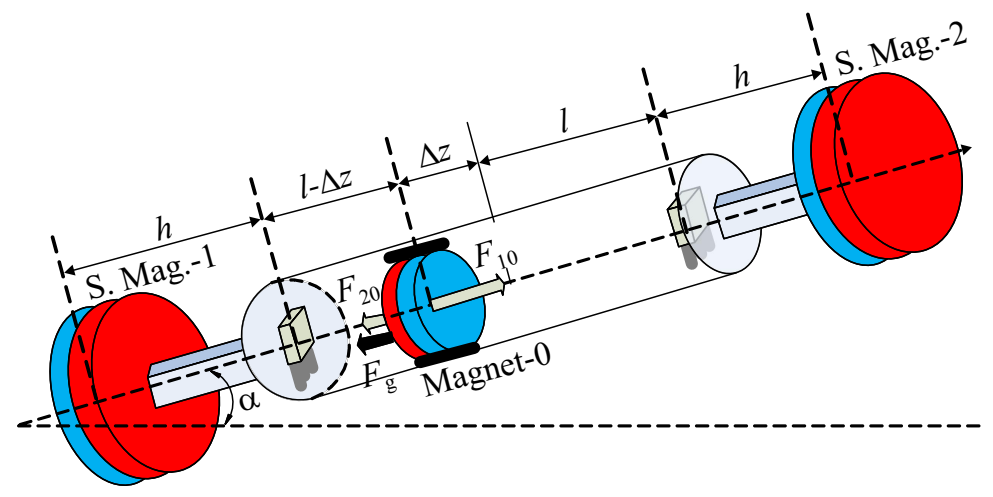

Fig. 3. Force diagram along $z$ axis for the sensor with a tilt angle $\alpha$

field of a magnet in the z-direction given in (1) can also be expressed as a simple power law $B(z)=A z^{-n}[2,4]$. Therefore, the potential energy can also be expressed in the form of $U=U_{0} z^{-n}$. By using this approach, it is possible to calculate the force between two magnets as shown in Fig. 2. The force applied to magnet-0 (that we assume to have uniform magnetization $M o$ and volume $V)$ in the $z$-direction due to the $B_{1}$ magnetic field formed by magnet-1 can be expressed by the gradient of the potential energy

$$
\begin{aligned}
F(z)=-\frac{\partial U}{\partial z} \hat{z} & =n U_{0} z^{-(n+1)} \hat{z}= \\
& -\iiint\left(M_{0} \frac{\mathrm{d} B_{1}}{\mathrm{~d} z} \hat{z}\right) \mathrm{d} V \cong M_{0} V \frac{\mathrm{d} B_{1}}{\mathrm{~d} z} \hat{z} .
\end{aligned}
$$

The forces acting on the mobile magnet (magnet- 0 ) along the z-direction are illustrated in Fig. 3. The $F_{01}$ force is applied by S. Mag. 1 and $F_{02}$ is applied by S.Mag.2 to balance and restore magnet- 0 . While the designed tilt sensor shown in Fig. 3 is in equilibrium $(\alpha=0)$, magnet0 will be at $\Delta z=0$ position with the effect of these forces. However, when the system is tilted with an $\alpha$ angle magnet-0 will be displaced because of the force component of the gravitation along the $\mathrm{z}$ direction. The equation of $F_{01}$ and $F_{02}$ forces can be obtained by using (2) and the Taylor expansions of these forces are expressed as follows

$$
\begin{aligned}
& F_{01}=n U_{0}(h+l-\Delta z)^{-(n+1)}= \\
& n U_{0}(h+l)^{-(n+1)}+\frac{1}{2} n(n+1) U_{0}(h+l)^{-(n+2)} \Delta z+ \\
& \frac{1}{6} n(n+1)(n+2) U_{0}(h+l)^{-(n+3)} \Delta z^{2}+\ldots
\end{aligned}
$$

$$
\begin{aligned}
& F_{02}=n U_{0}(h+l+\Delta z)^{-(n+1)}= \\
& n U_{0}(h+l)^{-(n+1)}-\frac{1}{2} n(n+1) U_{0}(h+l)^{-(n+2)} \Delta z+ \\
& \quad+\frac{1}{6} n(n+1)(n+2) U_{0}(h+l)^{-(n+3)} \Delta z^{2}+\ldots
\end{aligned}
$$

The force component along the $\mathrm{z}$ direction originating from gravity will be $F_{\mathrm{g}}=m g \sin (\alpha)$. The frictional force is neglected due to the lubrication effect of ferrofluid. Using (3) and (4) and ignoring the higher order terms, the expression of $\sin (\alpha)$ from the forces acting on the magnet- 0 can be written as follows

$$
\sin \alpha=\frac{F_{01}(z)-F_{02}(z)}{m g} \cong \frac{n(n+1) U_{0}(h+l)^{-(n+2)}}{m g} \Delta z
$$

It will be useful to rewrite (5) and obtain the displacement $\Delta z$ in terms of tilt angle

$$
\Delta z \cong \frac{m g(h+l)^{(n+2)}}{n(n+1) U_{0}} \sin \alpha .
$$

A similar relation between $\Delta z$ and $\alpha$ was defined and the mechanical sensitivity $(\Delta z / \alpha)$ examined for the small tilt angles $(\sin \alpha \approx \alpha)$ at the works of $\mathrm{Su}$ et al [2]. It is easy to see that maximum change of the displacement and the mechanical sensitivity will be at around $90^{\circ}$ tilt angle

It is needed to calculate magnetic fields on sensors to evaluate sensor responses. By using the same procedure calculating the forces, the magnetic field of magnets on the Hall sensors can be expressed in terms of displacement and distances from the Hall sensors as

$$
\begin{aligned}
& B_{h 1}=A_{s} h^{-n}+A_{s}(2 l+h)^{-n}-A_{0}(l-\Delta z)^{-n}, \\
& B_{h 2}=-A_{s} h^{-n}-A_{s}(2 l+h)^{-n}+A_{0}(l+\Delta z)^{-n} .
\end{aligned}
$$

The magnetic fields on the first and second Hall sensors were expressed as $B_{h 1}$ and $B_{h 2}$ respectively. (7) and (8) are written for the ideal case where the magnets are placed symmetrically at equal distances and the magnetization values of the side magnet sets are equal. Therefore, the $A_{s}$ and $A_{0}$ terms in $s$ have constant values which depend on magnetization and the dimensions of the magnets.

For ideal and identical linear Hall sensors, the voltage output will be proportional to the magnetic field of $B_{h}$ parallel to its surface normal. If this proportionality constant is called $k$, the $V_{h}$ voltages read by the linear Hall sensors with 2.5 null voltage can be expressed as

$$
V_{h}=2.5+k B_{h} .
$$




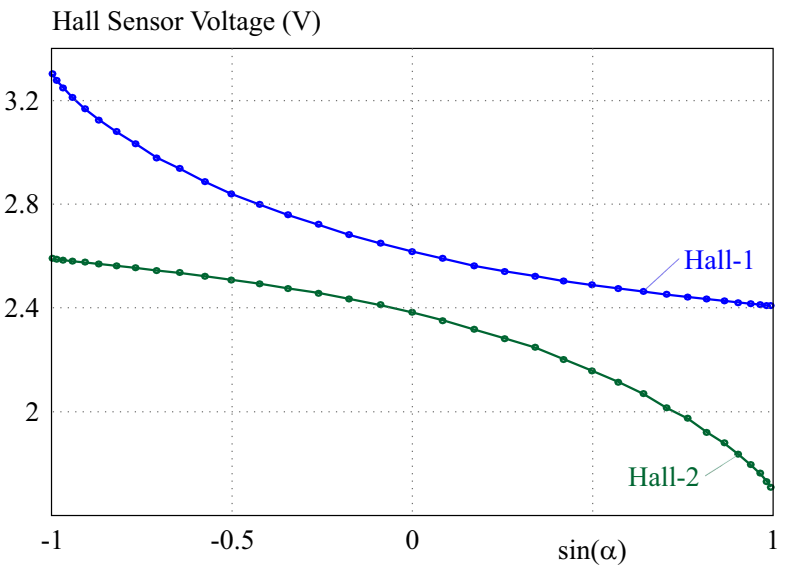

Fig. 4. Relation curves between hall sensor voltage values and $\sin \alpha$ for s.mag. $\mathrm{x} 3$

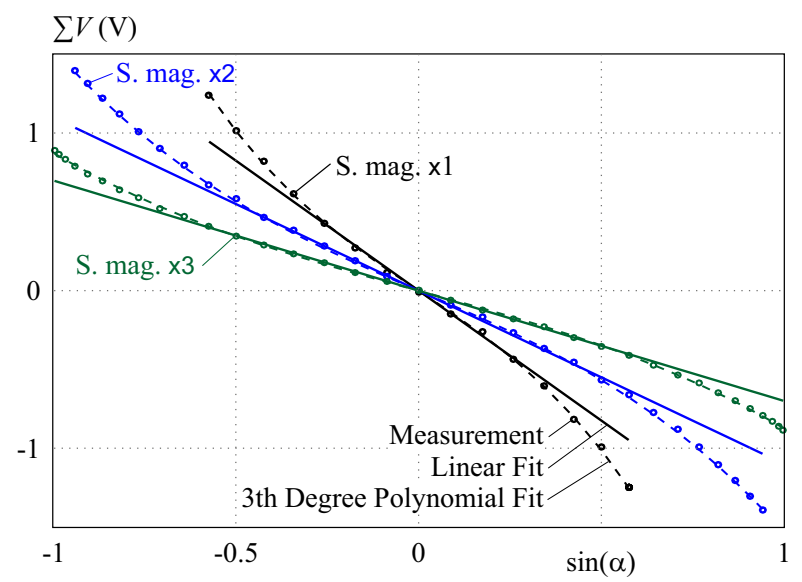

Fig. 5. Relation curves between the hall sensors voltage sum $(\Sigma V)$ values and $\sin (a)$ for 3 different number of the side magnets

Therefore the sum and difference of the Hall sensor output voltages by using (7) and (8) can be expressed as follows

$$
\begin{aligned}
V_{h 1}+V_{h 2}= & 5+k\left(B_{h 2}+B_{h 1}\right)= \\
& 5+k\left[A_{0}(l+\Delta z)^{-n}-A_{0}(l-\Delta z)^{-n}\right],
\end{aligned}
$$

$$
\begin{array}{r}
V_{h 2}-V_{h 1}=k\left(B_{h 2}-B_{h 1}\right)=k\left[-2 A_{s} h^{-n}-2 A_{s}(2 l+h)^{-n}\right. \\
\left.+A_{0}(l+\Delta z)^{-n}+A_{0}(l-\Delta z)^{-n}\right] .
\end{array}
$$

The sum and difference of voltage values given in (10) and (11) can be further simplified by using the Taylor expansion of these for $\Delta z$ and neglecting all terms of an order higher than 3

$$
\begin{gathered}
\Sigma V=V_{h 1}+V_{h 2} \cong 5+k A_{0} n(l)^{-n+1} \Delta z, \\
\Delta V=V_{h 2}-V_{h 1} \cong k\left[C+(2 / 6) A_{0} n(n+1)(l)^{-n+2} \Delta z^{2}\right] .
\end{gathered}
$$

All the terms independent of the displacement $\Delta z$ is considered as a constant $\mathrm{C}$ in (13). It was found from force expression (6) that $\Delta z$ is an approximate linear function of $\sin \alpha$. In this case, (12) and (13) will be as follows by using (6)

$$
\Sigma V=V_{h 1}+V_{h 2} \cong 5+k \frac{(l)^{-n+1}(h+l)^{n+2}}{n+1} \frac{m g A_{0}}{U_{0}} \sin \alpha,
$$

$$
\begin{aligned}
& \Delta V=V_{h 2}-V_{h 1} \\
& \quad \cong k\left[C+\frac{2(l)^{-n+2}(h+l)^{(2 n+4)}}{6 n(n+1)} \frac{(m g)^{2} A_{0}}{U_{0}^{2}} \sin ^{2} \alpha\right] .
\end{aligned}
$$

The sum of the Hall voltage is found to be linearly dependent on $\sin \alpha$, while the difference of the Hall voltage is quadratically dependent on $\sin \alpha$ for the small displacement of the mobile magnet. The distances between system elements are important parameters influencing the sensitivity of the system. Here in (14) and (15), the coefficients $U_{0}$ and $A_{0}$ are both linear functions of the magnetization value of the magnet- 0 and $U_{0}$ also depends on the magnetization of the side magnets. Due to this, the measurement sensitivity of the quadratic mode is inversely proportional to the magnetization of the magnets and proportional to the square of the magnet-0 mass. Besides that, the linear mode sensitivity mainly depends on the magnet-0 mass, and is inversely proportional to the magnetization of the side magnets. The maximum sensitivity of the system can be determined by differentiating (14) and (15) with respect to $\alpha$. Therefore, the maximum sensitivities are expected at the $45^{\circ}$ inclination angle for quadratic mode, and $90^{\circ}$ for the linear mode.

\section{Results and discussion}

Experiments were conducted using the system shown in Fig. 1 and Fig. 3. Measurements were taken separately with the system with the three different sets of side magnets. The peak value of the Hall sensors voltages would be at the inclination angle of $\pm 90^{\circ}$ with respect to ground. Owing to this, the gauge of rotation stage was set to $\pm 90^{\circ}$ when maximum values were read. The experiments were conducted by starting at $+90^{\circ}$ and measurements were taken with the tilt angle steps of $10^{\circ}$. The experiments were named according to the number of magnets on each side. For example, it was named as S.mag. x2 for the sets with two magnets. The measurements were first taken by placing 3 magnets on each side. Then, the number of magnets was reduced on both sides and the measurements were repeated.

In order to show the Hall sensor outputs due to the deviation of the magnet- 0 from its equilibrium position $(\Delta z=0)$ by tilt angle, data of one of the measurements was presented. Figure 4 illustrates the voltage output of each sensor with respect to the tilt angles between $+90^{\circ}$ and $-90^{\circ}$ for the system with the sets of 3 side magnets (S.mag. x3) . Although the Hall sensors chosen were identical their sensitivities were different. Therefore the voltage outputs of the Hall- 1 at $+90^{\circ}$ and the Hall- 2 at $-90^{\circ}$ were equalized by multiplying the Hall-2 sensor values by 


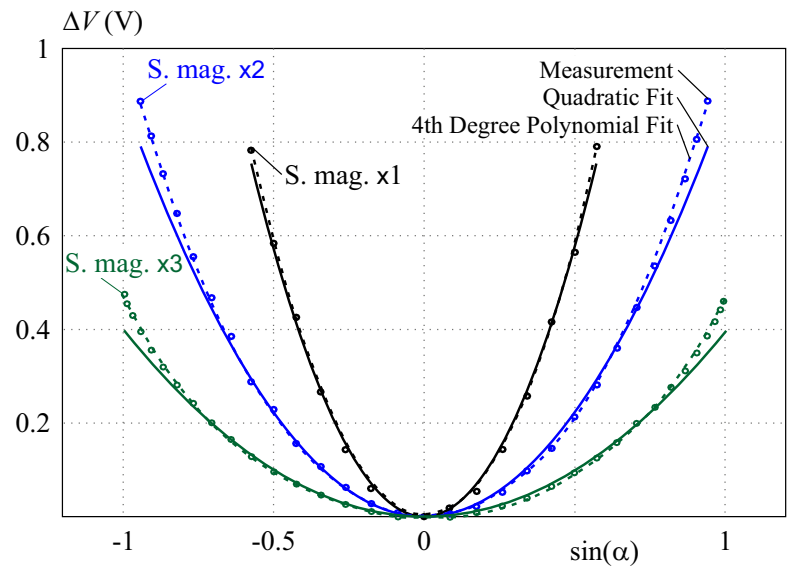

Fig. 6. Relation curves between the voltage difference of the hall sensors $(\Delta V$ and $\sin \alpha)$ for 3 different configuration of side magnets

Table 1. Fit functions and coefficients with $95 \%$ confidence bound

\begin{tabular}{lccc}
\hline$(\Sigma V)$ & $\Sigma V=s 1 \sin (\alpha)$ & \multicolumn{2}{c}{$\Sigma V=c 1 \sin (\alpha)+c 2 \sin ^{3}(\alpha)$} \\
& $\mathrm{s} 1$ & $\mathrm{c} 1$ & $\mathrm{c} 2$ \\
\hline S.mag. x1 & -1.68 & -1.55 & -1.91 \\
S.mag. x2 & -1.10 & -1.00 & -0.54 \\
S.mag. x3 & -0.70 & -0.63 & -0.24 \\
\hline$(\Delta V)$ & $\Delta V=q 1 \sin ^{2}(\alpha)$ & $\Delta V=q 1 \sin ^{2}(\alpha)+q 2 \sin ^{4}(\alpha)$ \\
\hline & $\mathrm{q} 1$ & $\mathrm{q} 1$ & $\mathrm{q} 2$ \\
S.mag. x1 & 2.30 & 2.18 & 0.69 \\
S.mag. x2 & 0.90 & 0.82 & 0.21 \\
S.mag. x3 & 0.40 & 0.34 & 0.13 \\
\hline
\end{tabular}

1.15. The linear range of the Hall sensors $(0.9-4.1 \mathrm{~V})$ was not exceeded at our results as seen in Fig. 4. For large tilt angles, the gravitation force component along the axis of the tilt system increases and causes magnet- 0 to get near to one of the Hall sensors. In accordance with expressions given before, the variations of the sensor outputs are getting larger via increment of the tilt angles.

The compatibility between experimental results and theoretical calculations of sum $\Sigma V$, and difference $\Delta V$ of the Hall sensors voltages were investigated. First, the results of the sum voltages $\Sigma V$ with respect to $\sin \alpha$ for the different numbers of the side magnet sets were shown in Fig. 5. The constant value of around $5 \mathrm{~V}$ due to the characteristic null voltage of the Hall sensors was subtracted from experimental data for simplification. The curves of linear and 3th degree polynomial fit to experimental data were presented. As shown in Fig. $5 \Sigma V$, values are close to the linear function of $\sin \alpha$ for relatively small tilt angles as expressed in (14). Since 3th and higher order terms were ignored while calculating (14) and (5), for higher values of tilt angle, these terms become dominant and $\Delta V$ deviates from the linear curve. Another deduction from these results is as the number of side magnets decreases, the sensitivity of the system increases at the cost of decrease of the linear range.
The results of the Hall sensors voltage difference $\Delta V$ with respect to $\sin \alpha$ for 3 different number of side magnet sets were shown in Fig. 6. Quadratic and 4th degree polynomials were fit to experimental data and resulting curves were presented. The $\Delta V$ expressed in (15) is a quadratic function of the tilt angle since 4th and higher order terms were neglected for relatively small angles. As can be seen from Fig. 6, curves deviate from the quadratic curve when these neglected higher order terms become dominant.

The effect of the number of magnets in combined identical magnets on their magnetization is relatively small. However, their combined magnetic field is still higher than single one due to the increase of the effective length $(a)$ as shown in (1). As a result of this increase in the magnetic field of the side magnets the force applied on the magnet- 0 increases. This force limits the displacement of the magnet- 0 and enlarges the measurement range of the designed system.

The data of $\Delta V$ and $\Sigma V$ were fitted with different sets of polynomial functions as illustrated in Fig. 5 and Fig. 6 . The coefficients of these polynomial functions estimated from the experimental data were given in Tab. 1 for different side magnet configurations. Linear and quadratic polynomial functions are in accordance with (14) and (15). Since these values were obtained approximately by series expansion and neglecting the 3rd and higher order terms, experimental data better fitted by including these terms. Coefficients of functions in Tab. 1 which are related to the system sensitivity decrease by the increase of the number of side magnets used. In linear range, one can use s1 coefficient in Tab. 1 as sensitivity by using the $\sin \alpha \approx \alpha$ approximation. In this case, the maximum linear sensitivity can be determined as $1.68 \mathrm{~V} / \mathrm{rad}$ for S.mag. $\mathrm{x} 1$ and minimum sensitivity can be determined as $0.7 \mathrm{~V} / \mathrm{rad}$ for S.mag. $\mathrm{x}$.

The results obtained illustrate that the system can work as a linear sensor for small inclination angles. Furthermore, it is shown that the sensor sensitivity and the measurement range can be arranged by changing the number of side magnets. Moreover, the dynamics of the sensor can be controlled by changing the other parameters given in (14) and (15).

\section{Conclusions}

In this study, an alternative bidirectional tilt sensor design which was constructed with simple components such as linear Hall effect sensors, ferrofluid and magnets were presented. The Hall sensor outputs depending on the displacement of the mobile magnet from the equilibrium position were recorded with respect to the tilt angles. As a result, it was shown that the system can be used as a linear and/or a quadratic tilt sensor at small angle values by calculating the sum and difference of two Hall sensors output. The agreement of the theoretical calculations with the data derived from the experimental results 
was obtained. It was also shown that the sensor sensitivity and the measurement range can be arranged by changing the number of balancing magnets used on the sides. The sensitivity values for the linear mode were calculated as approximately $0.7,1.1$ and $1.68 \mathrm{~V} / \mathrm{rad}$ for the three different balance magnet sets.

In addition, it is worth to mention that the use of the presented results is not limited to tilt measurement only. It is quite easy to develop a displacement or pressure sensing system by using the results of this study and the system. This system can be improved and adapted to many systems; moreover, it can be used in both undergraduate and graduate school labs for similar experiments.

\section{REFERENCES}

[1] J. Lenz and S. Edelstein, "Magnetic Sensors Their Applications", IEEE Sensors Journal, vol. 6 no. 3, 2006, pp. 631-649.

[2] R. Olarua and D. D. Dragoi, "Inductive Tilt Sensor with Magnets Magnetic Fluid", Sensors Actuators A, vol. 120, 2005, pp. $424-428$.

[3] O. Baltag, D. Costandache, and A. Salceanu, "Tilt Measurement Sensor", Sensors Actuators, vol 81, 2000, pp. 336-339.

[4] S. Su, D. Li, N. Tan, and G. Li, "The Study of a Novel Tilt Sensor Using Magnetic Fluid Its Detection Mechanism", IEEE Sensors Journal, vol. 17 no. 15, 2017, pp. 4708-4715.

[5] S. Das and C. Badal, "A Liquid Pendulum Based Optical Tilt Sensor", Sensors Actuators A: Physical, vol. 285, 2019, pp. 543-549.

[6] S. M. Khan, N. Qaiser, and M. M. Hussain, "An Inclinometer Using Movable Electrode In a Parallel Plate Capacitive Structure", AIP Advances, vol. 9 no. 4, 2019, pp. 045118.

[7] L. S. Sundar, P. Bhramara, N. T. R. Kumar, M. K. Singh, and A. C. M. Sousa, "Experimental Heat Transfer", International Journal of Heat Mass Transfer, vol. 109, 2017, pp. 440-453.
[8] R. E. Rosensweig, "Magnetic Fluids", Scientific American, vol. 247 no. 4, pp. 136-145, 1982.

[9] J. Yao, S. Liu, Z. Li, and D. Li, "A Novel Ferrofluid Inclinometer Exploiting a Hall Element", IEEE Sensors Journal, vol. 16 no. 22, 2016, pp. 7986-7991.

[10] U. Ausserlechner, "A Theory Of Magnetic Angle Sensors with Hall Plates without Fluxguides", Progress Electromagnetics Research B, vol. 49, 2013, pp. 77-106.

11] K. V. Dimitrov, "A 3-D Hall Sensor for Precise Angular Position Measurements", Turkish Journal of Physics, vol. 31 no. 2, 2007, pp. 97-101.

[12] M. I. González, "Forces Between Permanent Magnets: Experiments Model", Eur. J, Phys, vol. 38, 2017, pp. 025202.

[13] L. E. Gayetsky and C. L. Caylor, "Measuring the Forces Between Magnetic Dipoles", The Physics Teacher, vol. 45, 2007, pp. 348-351.

Received 31 May 2019

Yavuz Öztürk, born in 1978, received the MSc degree from University of Ege Izmir, Turkey at the Department of Electrical and Electronics Engineering in 2003. He received his PhD from this university in 2010 and became an Assistant Professor in 2011. During the period of 2010-2011 he worked at Université du Québec as a postdoctoral fellow on ultrafast optics. His main scientific interest includes magnetic materials and optical sensors.

Ismail Yarici, born in 1989, He received the BE, ME, in Electrical \& Electronics Engineering, Izmir Institute of Technology (IZTECH), Turkey, in 2012, and Ege University, Turkey, in 2015, respectively. He is in training for a doctor's degree in Electrical and Electronics Engineering on magnonphoton systems. Presently, he is research assistant at Department of Electrical and Electronics Engineering at University of Aydın Adnan Menderes, Turkey. 